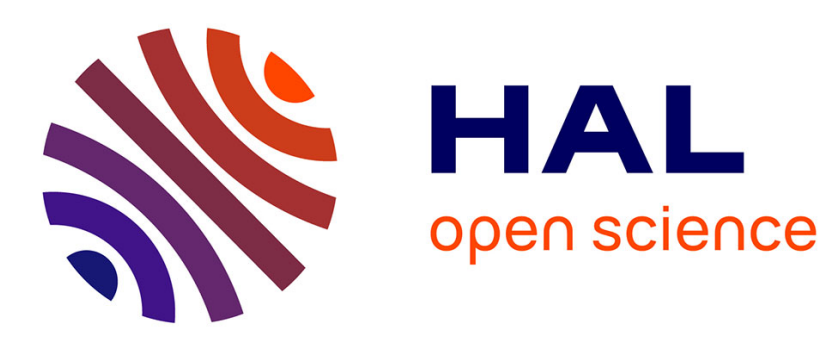

\title{
Continuum modelling of piezoelectromechanical truss beams: an application to vibration damping
}

Francesco Dell'Isola, Stefano Vidoli

\section{To cite this version:}

Francesco Dell'Isola, Stefano Vidoli. Continuum modelling of piezoelectromechanical truss beams: an application to vibration damping. Archive of Applied Mechanics, 1998, pp.19. hal-00495542

\section{HAL Id: hal-00495542 \\ https://hal.science/hal-00495542}

Submitted on 28 Jun 2010

HAL is a multi-disciplinary open access archive for the deposit and dissemination of scientific research documents, whether they are published or not. The documents may come from teaching and research institutions in France or abroad, or from public or private research centers.
L'archive ouverte pluridisciplinaire HAL, est destinée au dépôt et à la diffusion de documents scientifiques de niveau recherche, publiés ou non, émanant des établissements d'enseignement et de recherche français ou étrangers, des laboratoires publics ou privés. 


\title{
Continuum modelling of piezoelectromechanical truss beams: an application to vibration damping
}

\author{
F. dell'Isola, S. Vidoli
}

Summary We call piezoelectromechanical (PEM) truss beam a truss modular beam coupled with a transmission electrical line when the coupling is obtained by piezoelectric actuators which act as bars in the module and as capacitances in the electrical line.

The truss module length is assumed negligible with respect to the considered wave lengths. The transmission electrical line is assumed continuously distributed along the truss beam.

Applying the method of virtual power as expounded in [2] we formulate a continuum model for PEM truss beams and we prove that there exists a critical value for the transmission electrical impedance in the neighborhood of which the electromechanical modal coupling is maximum and the possible electrical dissipation of mechanical energy is relevant.

Key words modular truss beam, electric transmission line, piezoelectromechanical coupling, vibration control

\section{1}

\section{Introduction}

When formulating a mathematical model for a truss modular beam two different approaches are possible:

i) in a refined description, the deformation of every element of the truss is accounted for,

ii) in a continuum one, the kinematic of the truss is described by displacement and rotation fields defined on a curve.

The first approach, when applied to technologically meaningful instances, often leads to oversized numerical problems and models not easy to handle. Therefore, the second one (see for instance [4], [5], [6], [9], [10]) has been proposed: the displacement and rotation fields describe a homogenized average displacement and rotation of the generic constituent module.

In this paper, using the method of virtual power [2], we formulate a continuum description for a piezoelectromechanical truss beam which accounts for its modular structure, and use it to propose a new vibration damping technique.

The typical module constituting the considered truss beam is composed:

1) by a pair of flat rigid diaphragms connected by a number of elastic bars (some of which are piezoelectric) constrained to remain straight and

2) by an electric net connected also with the piezoelectric actuators.

The electromechanical system we consider presents the following features:

Accepted for publication 1 June 1997

F. dell'Isola, S. Vidoli

Dipartimento di Ingegneria Strutturale e Geotecnica,

Università di Roma "La Sapienza",

Via Eudossiana 18, I-00184 Roma, Italia

S.V. was supported by a research contract of Agenzia Spaziale Italiana, issued under the scientific responsibility of Prof. A. Di Carlo. Both authors acknowledge his friendly and accurate criticism. 
- at least one of the bars of each module is a piezoelectric actuator,

- the electrical transmission line shows the same periodicity properties of the truss beam, so that every mechanical module corresponds to a modular electrical net,

- the piezoelectric actuators are connected to the electrical line so that their equivalent circuits are to be regarded as part of the modular electrical net.

One important assumption we apply in this paper concerns the electromechanical behavior of the piezoelectric actuators: following [7], [8], they are regarded as cylinders undergoing Saint-Venant deformations only. Then the results obtained in [7], [8] allow us to find the relationship between the tabular Voigt piezoelectric constants and the relevant piezoelectric stiffnesses and impedances. A Mathematica program, supplied in Appendix A, needed to be developed in order to use quoted results in the present situation.

The new structural system studied in this paper deserves, in our opinion, some interest. This is proved by the following results obtained for simple, but interesting, $2 \mathrm{D}$ and $3 \mathrm{D}$ module choices in which extension, bending and torsion are examined.

We have studied the effects of the variation of the transmission line impedance on the wave propagation speed and on the modal and frequency response of the structure; we find that there exists a critical range of inductance in which the electromechanical coupling is relevant. In this range:

- the displacement-electric potential waves propagate simultaneously with two close propagation speeds,

- the wave energy is shared in comparable proportions in mechanical and electrical parts.

Unfortunately, the electromechanical coupling range for bending and the modular transmission line introduced in the present paper lies in the neighborhood of infinity: the inductances necessary to insure electromechanical coupling are not technically feasible. As a consequence, the modal damping ratios for bending are typically $10^{3} \div 10^{4}$ times smaller than in extension or in torsion, under comparable conditions. In a forthcoming paper, we will consider an electrical line whose evolution equation is a PDE in which fourth order space derivative appears, so obtaining a more efficient modal coupling.

Having used MатнематісA, we dispose (in the cases of extension and torsion) of the explicit symbolic formulas expressing wave speeds and the norms of the associated wave forms versus the electrical transmission impedance. This analytical treatment becomes difficult when studying the modal analysis under given boundary conditions or in the case of bending wave.

For this reason we have used a standard numerical procedure to find:

- the bending wave dispersion formulae,

- the eigenfrequencies electrically insulated, the corresponding eigenfunctions and the frequency response operator $\mathscr{H}(j \omega)$ of the clamped-clamped PEM beam.

We remark, concerning the frequency response operator, that a partition of the set of impedance values is obtained, as proved by the plot of a norm of the $\mathscr{H}(j \omega)$ operator. In a range, the response operator yields for different forcing frequencies either a purely mechanical or a purely electrical response. Instead in a given interval, centered around what we call "maximal exchange value", the response operator yields only coupled electromechanical responses: the range of resonant forcing frequencies results to be also sensibly increased with respect to the purely mechanical one. This means that around the "maximal exchange value" for impedance it is possible to damp modular truss by dissipating electrical energy.

Our numerical computation shows that this phenomenon can be exploited in technologically relevant instances: indeed the available PZT materials and actuators seem to allow the damping of truss beams of technologically relevant size.

To our knowledge, a continuously distributed damping for a modular truss has never been proposed before (see [13]). It seems to us that such damping technique is more efficient than those already proposed in the literature, characterized by the use of actuators supplying concentrated forces.

\section{2}

\section{Identification procedure}

In [4] truss beams are modelled using a continuum description generalizing the standard Bernoulli-Navier theory. The main result of the cited paper, based on the identification of the mechanical power on the refined level with that on the continuum one, allows for the determination of the constitutive equations for the contact and inertial actions explicitly in terms of 
the mechanical properties in the module. The identification of the mechanical response of the truss modular beam, when modelled as a one-dimensional continuum, is then possible by means of an algorithmic procedure.

The same approach applied to the electrical transmission line leads to a standard homogenization procedure in which the electrical impedance is continuously distributed per unit length.

We adapt here the identification procedure proposed in [4] to piezoelectromechanical truss beams.

\section{1}

\section{Refined model}

We assume that:

- the bars are constrained to remain straight, therefore they can be only elongated,

- the electric potential is assumed linear in any piezoelectric bar (see [8]).

With these hypotheses, the state of each module is completely determined by the placement of the diaphragms which are assumed to be rigid, and by the capacitive charges at the extremes of the piezoelectric bars.

Therefore, let us assume as "kinematical descriptors" characterizing the state of the considered system, the position of a point in the diaphragm $X_{o}$, a triple of unit vectors $\mathbf{G}_{k}$ representing the attitude of the diaphragm, and the set of the charges at the ends of the piezoelectric bars $Q$.

We shall label with a superscript minus $(-)$ and plus $(+)$ two consecutive diaphragms belonging to a module. So, respectively, on the reference and actual configuration, the kinematical descriptors of a module are

$X_{o}^{ \pm}, \mathbf{G}_{k}^{ \pm}, Q^{ \pm}$

$x_{o}^{ \pm}, \mathbf{g}_{k}^{ \pm}, q^{ \pm}$

so that the positions of the end points of the typical bar in the module are

$x_{b}^{ \pm}=x_{o}^{ \pm}+\alpha_{b}^{ \pm} \mathbf{g}_{2}^{ \pm}+\beta_{b}^{ \pm} \mathbf{g}_{3}^{ \pm}$,

where $\alpha_{b}^{ \pm}, \beta_{b}^{ \pm}$are the time-independent coordinates of the bar labeled $b$ with respect to the vectors $\mathbf{g}_{2}$ and $\mathbf{g}_{3}$ in the diaphragm plane.

Corresponding to the previous choices, being

$e_{b}:=\frac{\left\|x_{b}^{+}-x_{b}^{-}\right\|}{L_{b}}-1$

the elongation of the bar, the time-rate deformation field in any bar is given by

$\dot{e}_{b}=\frac{\left(x_{b}^{+}-x_{b}^{-}\right) \overline{\left(x_{b}^{+}-x_{b}^{-}\right)}}{L_{b}\left\|x_{b}^{+}-x_{b}^{-}\right\|}$

where $L_{b}:=\left\|X_{b}^{+}-X_{b}^{-}\right\|$is the reference length of the bar.

Their electrical counterparts will be

$\rho_{b}:=q_{b}^{+}-q_{b}^{-}$

and the piezoelectric capacitive current

$\dot{\rho}_{b}=\frac{\cdot}{\left(q_{b}^{+}-q_{b}^{-}\right)}$.

For any module, the expression of the electrical power associated to the set $\mathscr{N}_{p}$ of its piezoelectric bars is

$\mathscr{W}_{e}^{\text {refined }}=\sum_{p \in \mathscr{N}_{p}} \phi_{p} \cdot \dot{\rho}_{p}$

where $\phi_{p}$ represents the electric potential difference between the end points of the piezoelectric bar $p$. 
In a similar way, for any module we express the mechanical power of the contact actions $n_{b}$

$\mathscr{W}_{c}^{\mathrm{refined}}=\sum_{b \in \mathscr{N}_{b}} n_{b} \cdot \dot{e}_{b}$

and of the inertial actions

$x(\xi):=x_{b}^{-}+\frac{\xi}{L_{b}}\left(x_{b}^{+}-x_{b}^{-}\right)$

$\mathscr{W}_{\text {in }}^{\text {refined }}=-\sum_{b \in \mathscr{N}_{b}} \int_{0}^{L_{b}} \delta_{b} \ddot{x}(\xi) \cdot \dot{x}(\xi) \mathrm{d} \xi-\sum_{d \in \mathcal{N}_{d}} \int_{0}^{L_{d}} \delta_{d} \ddot{x}(\xi) \cdot \dot{x}(\xi) \mathrm{d} \xi$

where $\mathscr{N}_{d}$ and $\mathscr{N}_{b}$ are, respectively, the set of the bars constituting and connecting the diaphragms and $\delta_{b}, \delta_{d}$ are the densities per unit length of the bars.

Concerning the refined constitutive relations for the contact actions and for the electric potentials, we limit our attention to the linear case. Following [7], [8] the electro-mechanical behavior of the piezoelectric actuators is derived. We regard them as Saint-Venant cylinders, starting from the equations for the Cauchy stress tensor $\mathbf{T}$ and the electric displacement tensor D

$\operatorname{div} \mathbf{T}=0, \quad \operatorname{div} \mathbf{D}=0$

The linear constitutive equations for piezoelectric materials can be found in [1]. Equations are solved under the Saint-Venant hypothesis in order to determine the electromechanical stiffnesses of such actuators. As our interest is limited to purely extensional actuators, we quote here the linear constitutive relations expressing the contact action and the electric potential as functions of the electromechanical deformation field

$n_{b}=k_{e e} e_{b}+k_{e \rho} \rho_{b}$,

$\phi_{b}=k_{\rho e} e_{b}+k_{\rho \rho} \rho_{b}$.

The results of [7], [8] allow us to find the relationship between the tabular Voigt piezoelectric constants and the more technologically useful actuator constants $k_{e e}, k_{e \rho}, k_{\rho e}, k_{\rho \rho}$. A MATHEMATICA program devoted to transform the Voigt constants is given in Appendix A.

The constitutive relations for the purely electric elements (resistances and inductances) are written as

$\phi_{p}=k_{R} \dot{\rho}_{p}, \quad \phi_{p}=k_{L} \ddot{\rho}_{p}$.

\section{2}

\section{Continuum model}

The coarse model apt to describe our electromechanical system is a one-dimensional continuum endowed with structure: we assume as model for the mechanical part the standard Euclidean structure used in the theory of Bernoulli-Navier beam (see for a complete discussion [4]), and for the electric part the standard model from the theory of the continuous electrical transmission line. Finally, the electromechanical coupling will be modelled assuming that every used constitutive equation depends on all the electro-mechanical state parameters listed below.

The parameters describing the state of every material point $\mathscr{P}$ of this continuum are chosen paralleling the choices made in the refined model. Respectively, in the reference and actual configuration we have

$P_{o}(\varsigma), \mathbf{D}_{k}(\varsigma), \Gamma(\varsigma)$

$p_{o}(\varsigma, t), \mathbf{d}_{k}(\varsigma, t), \gamma(\varsigma, t)$, 
where $\varsigma$ and $t$ are the spatial and temporal coordinates, $p_{o}$ represents the position of the point $\mathscr{P}, \mathbf{d}_{k}$ the directors orienting $\mathscr{P}$ and $\gamma(\varsigma)=\left(\gamma_{1}(\varsigma), \gamma_{2}(\varsigma), \ldots\right)$ the charges stored in the piezoelectric components in the $[0, \varsigma]$ interval.

The generalized displacement field is described by the following relations:

$\mathbf{u}(\varsigma, t)=p_{o}(\varsigma, t)-P_{o}(\varsigma)$,

$\mathbf{R} \in$ Orth, $\mathbf{d}_{k}(\varsigma, t)=\mathbf{R}(\varsigma, t) \mathbf{D}_{k}(\varsigma)$,

$\delta(\varsigma, t)=\gamma(\varsigma, t)-\Gamma(\varsigma)$.

The velocity field is defined deriving with respect to $t$ the previous equations

$$
\begin{aligned}
\mathbf{w} & =\dot{\mathbf{p}}_{o}, \\
\mathbf{W} & =\dot{\mathbf{R}} \mathbf{R}^{T} \Rightarrow \dot{\mathbf{d}}_{k}=\mathbf{W d}_{k}, \\
l & =\dot{\gamma} .
\end{aligned}
$$

Finally, the generalized deformation field is chosen comparing, as usual, the actual and the reference configurations

$$
\begin{aligned}
& \mathbf{e}=\mathbf{R}^{T} p_{o}^{\prime}-P_{o}^{\prime}, \\
& \mathbf{E}=\mathbf{R}^{T} \mathbf{R}^{\prime}, \\
& \varrho=\gamma^{\prime}-\Gamma^{\prime} .
\end{aligned}
$$

Let us now write the expressions of mechanical and electrical power: the actions which expend power on the time-rate generalized deformation field are the Piola-Kirchhoff contact actions $\mathbf{s}, \mathbf{S}$ and the electric potential field $\varphi$. On a module of reference length $L$ we have

$$
\begin{aligned}
& \mathscr{W}_{c}^{\text {coarse }}=\int_{0}^{L}\left(\mathbf{s} \cdot \dot{\mathbf{e}}-\frac{1}{2} \mathbf{S} \cdot \dot{\mathbf{E}}\right) \mathrm{d} \varsigma, \\
& \mathscr{W}_{e}^{\text {coarse }}=\int_{0}^{L}(\varphi \cdot \dot{\varrho}) \mathrm{d} \varsigma,
\end{aligned}
$$

Finally, denoting by $(\mathbf{b}, \mathbf{B})$ the distributed inertial actions, the power expended is given by

$$
\mathscr{W}_{c}^{\text {coarse }}=\int_{0}^{L}\left(\mathbf{b} \cdot \mathbf{w}-\frac{1}{2} \mathbf{B} \cdot \mathbf{W}\right) \mathrm{d} \varsigma .
$$

The mechanical balance equations in terms of the Piola-Kirchhoff stress fields are

$$
\begin{aligned}
& (\mathbf{R s})^{\prime}+\mathbf{b}=0, \\
& \left(\mathbf{R S R}^{T}\right)^{\prime}+\mathbf{p}_{o}^{\prime} \wedge \mathbf{R s}+\mathbf{B}=0 .
\end{aligned}
$$

Now, let us choose the typical module for the electrical circuit, i.e. show how the piezoelectric bars are connected to each other as well as with the resistance and inductance elements. Figure 1 represents the more general mesh used in the present paper.

For a linear continuous transmission line characterized by the chosen electrical mesh, Kirchhoff circuital laws and constitutive equations read

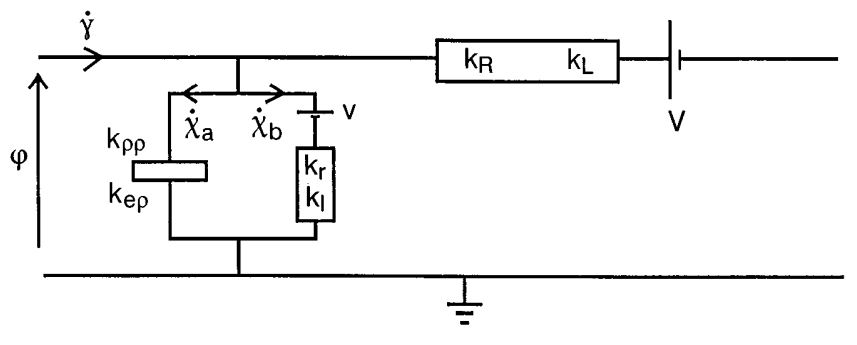

Fig. 1. Typical modular electric mesh constituting the transmission line. The first element from the left represents the capacitance of the PZT bar 
$\varphi^{\prime}=\kappa_{L} \ddot{\gamma}+\kappa_{R} \dot{\gamma}+V$

$\dot{\gamma}^{\prime}=\dot{\chi}_{a}+\dot{\chi}_{b}$,

$\kappa_{\rho \rho} \chi_{b}+\kappa_{\rho e} u^{\prime}=\varphi$

$\kappa_{l} \ddot{\chi}_{a}+\kappa_{r} \dot{\chi}_{a}=v+\varphi$

where constitutive equations (21) and (22) are the continuum counterpart of (9) and (10). For vanishing initial conditions we get

$\chi_{a}(t)=\frac{1}{\kappa_{l}} \int_{0}^{t} \int_{0}^{t^{\prime}} \exp ^{-\frac{\kappa_{r}}{\kappa_{l}}\left(t^{\prime}-\tau\right)}(v+\varphi)(\tau) \mathrm{d} \tau \mathrm{d} t^{\prime}$

Given a function $f=f(\varsigma, t)$, let us introduce the following integral operator

$\mathscr{I}_{\alpha}(f):=\int_{0}^{t} \int_{0}^{t^{\prime}} \exp ^{-\alpha\left(t^{\prime}-\tau\right)} f(\cdot, \tau) \mathrm{d} \tau \mathrm{d} t^{\prime}$

With the following two properties

$\lim _{\alpha \rightarrow \infty} \mathscr{I}_{\alpha}(f)=0$,

$\left[\mathscr{I}_{\alpha}(f)\right]^{\prime}=\mathscr{I}_{\alpha}\left(f^{\prime}\right)$,

so that

$\chi_{a}=\frac{1}{\kappa_{l}} \mathscr{I}_{\frac{\kappa_{r}}{\kappa_{l}}}(v+\varphi)$

With these assumptions

$\varphi^{\prime}=\kappa_{R} \dot{\gamma}+\kappa_{L} \ddot{\gamma}+V$

$\kappa_{\rho \rho} \gamma^{\prime}+\kappa_{\rho e} u^{\prime}=\varphi+\frac{\kappa_{\rho \rho}}{\kappa_{l}} \mathscr{I}_{\frac{\kappa_{r}}{\kappa_{l}}}(v+\varphi)$.

The previous equations are easily rearranged in terms of $\gamma$ to give

$\kappa_{\rho \rho} \gamma^{\prime \prime}+\kappa_{\rho e} u^{\prime \prime}=\kappa_{L} \ddot{\gamma}+\kappa_{R} \dot{\gamma}+V+\frac{\kappa_{\rho \rho}}{\kappa_{l}} \mathscr{I}_{\frac{\kappa_{r}}{\kappa_{l}}}\left(v^{\prime}+\kappa_{R} \dot{\gamma}+\kappa_{L} \ddot{\gamma}+V\right)$,

which for $\kappa_{r} \rightarrow \infty\left(\mathscr{I}_{\frac{K_{r}}{\kappa_{l}}} \rightarrow 0\right)$ reduces to a wave equation for the charge vector density $\gamma$.

We note that for $\kappa_{R} \rightarrow \infty$, Eq. (19) reduces to

$\dot{\gamma}=0$.

Then $\gamma(\cdot, t)=\gamma_{0}$ and $\dot{\gamma}^{\prime}=0$, so that Eqs. (20), (21) and (22) become

$\dot{\chi}_{a}=-\dot{\chi}_{b}=: \dot{\chi}$

$\kappa_{\rho \rho} \gamma_{0}^{\prime}-\kappa_{\rho \rho} \chi+\kappa_{\rho e} u^{\prime}=\varphi$,

$\kappa_{l} \ddot{\chi}+\kappa_{r} \dot{\chi}=v+\varphi$,

which reduce to the Kirchhoff laws for the RLC circuit separately connected to every piezoelectric bar (remark) that in this limit case $\gamma_{0}^{\prime}$ represents the initial distribution of capacitive charge:

$\kappa_{l} \ddot{\chi}+\kappa_{r} \dot{\chi}+\kappa_{\rho \rho}\left(\chi-\gamma_{0}^{\prime}\right)-\kappa_{\rho e} u^{\prime}=v$. 


\section{Constitutive relations}

A relation between the refined and the continuum model is established by assigning a map from the local placement in the coarse model $\left(p_{o}, \mathbf{d}_{\mathbf{k}}, \gamma\right)$ to the local placement of a module in the refined model $\left(x_{o}^{ \pm}, \mathbf{g}^{ \pm}, q^{ \pm}\right)$

$$
\begin{array}{ll}
x_{o}^{-}=p_{o}-\frac{L}{2} p_{o}^{\prime}, & x_{o}^{+}=p_{o}+\frac{L}{2} p_{o}^{\prime}, \\
\mathbf{g}_{k}^{-}=\mathbf{d}_{k}-\frac{L}{2} \mathbf{d}_{k}^{\prime}, & \mathbf{g}_{k}^{+}=\mathbf{d}_{k}+\frac{L}{2} \mathbf{d}_{k}^{\prime}, \\
q^{-}=\gamma-\frac{L}{2} \gamma^{\prime}, & q^{+}=\gamma+\frac{L}{2} \gamma^{\prime},
\end{array}
$$

denoting by $L$ the reference length of the module.

In order to identify the continuum constitutive functions we assume that, for any pair of corresponding motions, the power expended by the actions prescribed within the fine model equals the power expended by the corresponding actions within the coarse model

$$
\begin{aligned}
& \mathscr{W}_{c}^{\text {coarse }}=\mathscr{W}_{c}^{\text {refined }}, \\
& \mathscr{W}_{e}^{\text {coarse }}=\mathscr{W}_{e}^{\text {refined }}, \\
& \mathscr{W}_{\text {in }}^{\text {coarse }}=\mathscr{W}_{\text {in }}^{\text {refined }}
\end{aligned}
$$

This identification procedure was used by [4] in a purely mechanical contest, and has shown its validity in the study of technical problems [9], [10]. Moreover, the results expounded in [2] clearly indicate that, when dealing with the electromechanical coupling phenomena, the most useful tool is given by the method of virtual power: we are confident that the identities (32) allow among the possible continuum models the determination of that one which better approximates the discrete refined model.

By using explicitly the expressions (4), (5), (14), (15) for power, and by a massive use of computer aided symbolic calculation via Eqs. (31), we finally get the constitutive coarse relations for the contact actions $(\mathbf{s}, \mathbf{S})$ :

$$
\begin{aligned}
& \mathbf{s}=\sum_{b \in \mathscr{N}_{b}} \mathbf{s}_{b} ; \quad \mathbf{s}_{b}:=\mathbf{R}_{b}^{T} \frac{\left(x_{b}^{+}-x_{b}^{-}\right)}{\left\|x_{b}^{+}-x_{b}^{-}\right\|} n_{b}, \\
& \mathbf{s}=\sum_{b \in \mathscr{N}_{b}} \mathbf{s}_{b} ; \quad \mathbf{s}_{b}:=\frac{1}{2}\left[\left(\alpha_{b}^{-}+\alpha_{b}^{+}\right) \mathbf{D}_{2}+\left(\beta_{b}^{-}+\beta_{b}^{+}\right) \mathbf{D}_{3}\right] \wedge \mathbf{s}_{b},
\end{aligned}
$$

and for the electrical and piezoelectrical ones:

$$
\begin{aligned}
\kappa_{\rho e}=k_{\rho e}, & \kappa_{\rho \rho}=k_{\rho \rho} L, \\
\kappa_{R}=\frac{k_{R}}{L}, & \kappa_{L}=\frac{k_{L}}{L} .
\end{aligned}
$$

Equations (34) represent a simple homogenization of the refined discrete parameters $(k .$.$) in$ the coarse continuum ones $(\kappa .$.$) used in the classical theory of continuously distributed$ transmission lines.

We refer to the Appendix B for a complete explicit expression of the non-linear inertial constitutive coarse relations.

\section{3}

\section{Linearized equations: applications to particular truss modules}

Equations (33), (34) and (46 through 56) in Appendix B provide the linear expressions for the piezo electrical and purely electrical actions and the nonlinear ones for the contact and inertial actions. In order to start the analysis of the behavior of PEM beams, we have linearized these last ones (see in Appendix $\mathrm{C}$ for details of such a linearization) assuming that either $\kappa_{r} \rightarrow \infty$ or $\kappa_{R} \rightarrow \infty$.

Under the assumption $\kappa_{r} \rightarrow \infty$, we introduce the linearized expressions of $(\mathbf{s}, \mathbf{S})$ and $(\mathbf{b}, \mathbf{B})$ in the balance equations $(17,18)$, and rearrange the mechanical parameters in a function vector $\mathbf{u}(\varsigma, t)$ and the electrical ones in $\gamma(\varsigma, t)$. For example, in the case of a $2 \mathrm{D}$ truss beam with one 
piezoelectric bar per module the function vector $\mathbf{u}(\varsigma, t)$ contains the axial displacement $u$, the transverse displacement $v$ and the rotation fields $\vartheta$

$\mathbf{u}(\varsigma, t)=\left(\begin{array}{c}u(\varsigma, t) \\ v(\varsigma, t) \\ \vartheta(\varsigma, t)\end{array}\right)$

while $\gamma(\varsigma, t)$ is a scalar function representing the capacitive charge stored in segment $[0, \varsigma]$ of the line. The linear equations of the considered electromechanical system may be written now as follows:

$$
\begin{aligned}
& \mathscr{K}_{e e}^{(0)} \mathbf{u}+\mathscr{K}_{e e}^{(1)} \mathbf{u}^{\prime}+\mathscr{K}_{e e}^{(2)} \mathbf{u}^{\prime \prime}+\mathscr{K}_{e \rho} \gamma^{\prime \prime}+\mathscr{M}^{(0)} \ddot{\mathbf{u}}+\mathscr{M}^{(1)} \ddot{\mathbf{u}}^{\prime}+\mathscr{M}^{(2)} \ddot{\mathbf{u}}^{\prime \prime}=0, \\
& \mathscr{K}_{\rho \rho} \gamma^{\prime \prime}+\mathscr{K}_{\rho e} \mathbf{u}^{\prime \prime}+\mathscr{K}_{R} \dot{\gamma}+\mathscr{K}_{L} \ddot{\gamma}=V,
\end{aligned}
$$

where $\mathscr{K}^{(. .)}, \mathscr{M}^{(.)}, \mathscr{K}_{R}$ and $\mathscr{K}_{L}$, are, respectively, the stiffness, mass and impedance matrices, whose expression in term of the electromechanical properties of the module are known.

Similarly, under the assumption $\kappa_{R} \rightarrow \infty$, we have

$\mathscr{K}_{e e}^{(0)} \mathbf{u}+\mathscr{K}_{e e}^{(1)} \mathbf{u}^{\prime}+\mathscr{K}_{e \rho}^{(2)} \mathbf{u}^{\prime \prime}+\mathscr{K}_{e \rho} \chi^{\prime}+\mathscr{M}^{(0)} \ddot{\mathbf{u}}+\mathscr{M}^{(1)} \ddot{\mathbf{u}}^{\prime}+\mathscr{M}^{(2)} \ddot{\mathbf{u}}^{\prime \prime}=0$,

$\mathscr{K}_{\rho \rho} \chi+\mathscr{K}_{\rho e} \mathbf{u}^{\prime \prime}+\mathscr{K}_{r} \dot{\chi}+\mathscr{K}_{i} \ddot{\chi}=v$,

in which the variable $\chi$ plays the role of an internal variable (see [3]).

\section{1}

\section{Description of the considered truss module}

We have examined three different truss modules.

In the first one, shown in Fig. 2, there is only one piezoelectric bar centered along the symmetry axis; in addition, all the geometrical and mechanical properties are symmetric with respect to this axis. This fact allows us to obtain the axial displacement and electric current equations uncoupled from the shear and bending ones. This is a particular case of Eqs. (35) in which the stiffness, mass and impedance matrices reduce to scalar numbers: wave analysis can be easily faced in a symbolic way. The bars containing the piezoelectric actuators are marked with bold lines; the normal lines indicate the aluminium bars.

The second module, with four PZT bars electrically connected in parallel, is designed to couple torsion with the electric transmission line, and can be studied exactly in the same way as the first one.

The third module, with two symmetric piezoelectric actuators, is designed to establish coupling phenomena between the bending beam and the considered electric transmission line.

Table 1 resumes the geometrical and mechanical data of the described truss modules. In all the modules we assume as piezoelectric actuator a cylinder of PZT5 material whose constants are listed in Appendix A.

\section{4}

\section{Analysis of numerical results}

The numerical methods we use for the first analysis of Eqs. (35) are based on standard FE and Runge-Kutta discretizations, implemented by the computation environment Mathematica. The section is divided into three parts.

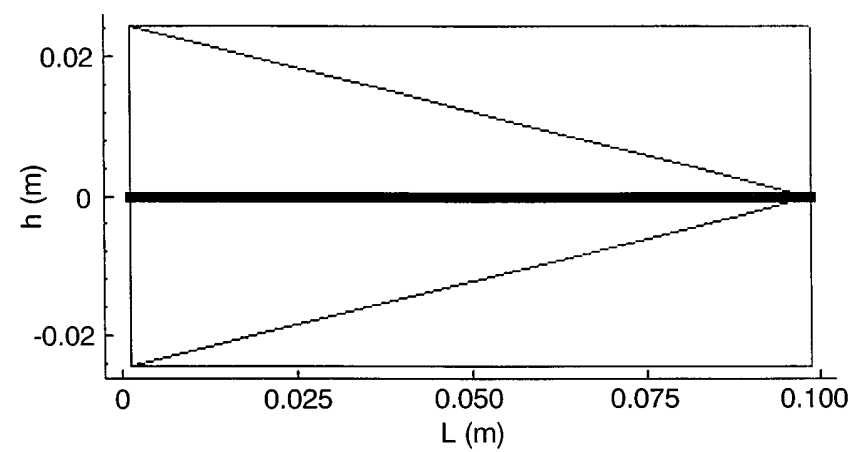

Fig. 2. First truss module (bold lines for PZT bars) 


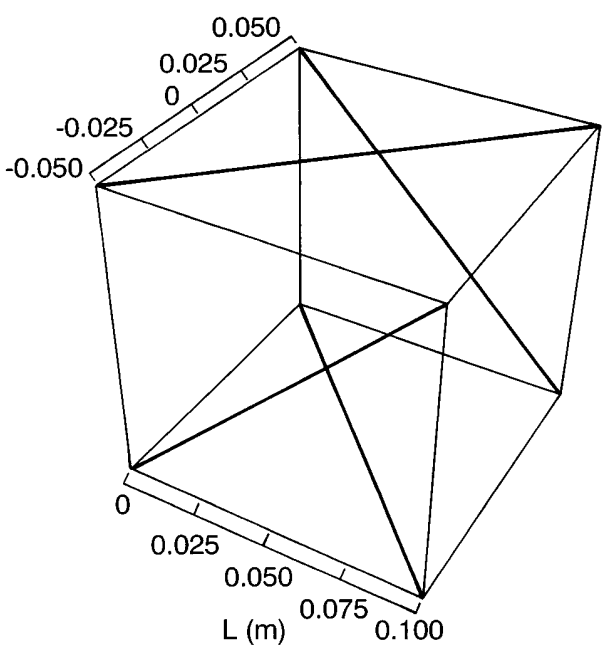

$\mathrm{L}(\mathrm{m})$
0.0750 .100

Fig. 3. Second truss module (bold lines for PZT bars)

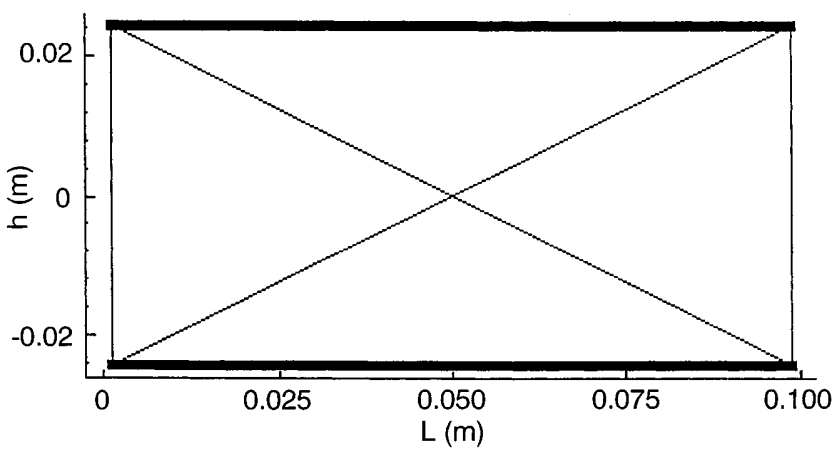

Fig. 4. Third truss module (bold lines for PZT bars)

In Sect. 4.1, we consider an infinite truss beam, and study the transversal, compression and torsional wave propagation, the dispersion formula and the electromechanical coupling for all the modules presented in Sect. 3.1.

In Sect. 4.2, we consider the finite clamped-clamped and electrically insulated electromechanical beam. The spectrum of the differential operator in (35) is discrete, and determined via the simultaneous diagonalization of the corresponding electromechanical rigidity and mass FEM matrices. Subsequently, we determine the set of eigenfunctions, each of which is composed by a purely mechanical and purely electrical part. Finally we determine, via a Fourier Transform of the FE discretized version of (35), its frequency response operator.

In Sect. 4.3, we introduce in the coupled transmission line a dissipative impedance. For all considered modules, we determine the modal damping ratio as a function of the line impedance. This leads us to consider damped vibration only for extensional and torsional problems. This is done projecting (35) on the first two eigenfunctions found in the previous Section, thus finding a second order system of ODEs for the corresponding coefficients. The Cauchy problem for this system of ODEs we solve using a standard Runge-Kutta integrator. Different values for impedance are considered. We find the range for impedance in which a relevant energy exchange between the mechanical and the electrical components occurs and an efficient electrical damping is possible.

Table. 1. Mechanical and geometrical properties of the aluminium and PZT bars

\begin{tabular}{lll}
\hline Geometry & PZT bar & Al bar \\
\hline$L=0.1 \mathrm{~m}$ & $\rho_{\mathrm{PZT}}=7000 \mathrm{~kg} / \mathrm{m}^{3}$ & $\rho_{\mathrm{Al}}=2800 \mathrm{~kg} / \mathrm{m}^{3}$ \\
$h=0.05 \mathrm{~m}$ & $A_{\mathrm{PZT}}=10^{-4} \mathrm{~m}^{2}$ & $A_{\mathrm{Al}}=2.510^{-5} \mathrm{~m}^{2}$ \\
& $l_{\mathrm{PZT}}=0.05 \mathrm{~m}$ & $G_{\mathrm{Y}, \mathrm{Al}}=70 \mathrm{GPa}$ \\
\hline
\end{tabular}


4.1

Wave analysis: dispersion formulae and propagation speeds

Wave analysis on Eqs. (35) is faced as usual, by searching solutions of the form

$$
\left(\begin{array}{l}
\mathbf{u}(\varsigma, t) \\
\gamma(\varsigma, t)
\end{array}\right)=\left(\begin{array}{l}
\overline{\mathbf{u}} \\
\bar{\gamma}
\end{array}\right) \cdot e^{\lambda \varsigma+j \omega t}
$$

The ratio $\omega / \lambda$ physically represents the wave speed; the ratio $\left|\overline{\mathbf{u}}_{j}\right| /\left|\bar{\gamma}_{j}\right|$ between two components of the mechanical and electrical eigenvectors measures electromechanical coupling in the wave.

We can analytically determine the explicit dispersion formula $\lambda=\lambda(\omega)$ for axial displacement-current and twist angle-current waves, arising when the modules in Fig. 2 and in Fig. 3 are considered. This can be done as in both cases in Eqs. (35) the stiffness, impedance and mass matrices are reduced to scalar numbers and the last two addends in Eqs (35) ${ }_{1}$ are vanishing. With the obvious meaning of the symbols we have

$$
\left(\frac{\lambda}{\omega}\right)^{2}=\sqrt{\frac{\kappa_{e e} \kappa_{L}+\kappa_{\rho \rho} m_{0} \pm \sqrt{\kappa_{e e}^{2} \kappa_{L}^{2}+4 \kappa_{e \rho} \kappa_{L} \kappa_{\rho e} m_{0}-2 \kappa_{e e} \kappa_{L} \kappa_{\rho \rho} m_{0}+\kappa_{\rho \rho}^{2} m_{0}^{2}}}{2\left(\kappa_{e e} \kappa_{\rho \rho}-\kappa_{\rho e} \kappa_{e \rho}\right)}} .
$$

The line inductance minimizing the difference between the two wave speeds and maximizing the electromechanical coupling is given by

$\overline{\kappa_{L}}=\frac{2 \kappa_{e \rho} \kappa_{\rho e}-\kappa_{\rho \rho} \kappa_{e e} \pm \sqrt{\left(2 \kappa_{e \rho} \kappa_{\rho e}-\kappa_{\rho \rho} \kappa_{e e}\right)^{2}-\kappa_{e e}^{2} \kappa_{\rho \rho}^{2}}}{\kappa_{e e}^{2}} \cdot m_{0}$.

In Fig. 5 are shown, for the first truss module, the variation of the wave speeds when increasing the line inductance $\kappa_{L}$, and the electromechanical coupling measured comparing the elastic and capacitive energy of the different waves.

We note that for both modules the existence of a critical value $\overline{\kappa_{L}}-$ which we will call "maximal exchange value" - for inductance is numerically proven. It maximizes the electromechanical coupling and minimizes the difference among the propagation speeds of those waves for which the electromechanical coupling is relevant. As one should expect, the maximal exchange value for inductance will play a relevant role also in the following modal analysis.

Unfortunately, the maximal exchange value for inductance, in the case of bending-current waves, is $+\infty$ for the present choice of the coupled transmission line; this is shown by the numerical determination of the dispersion formula and the corresponding study of electro-
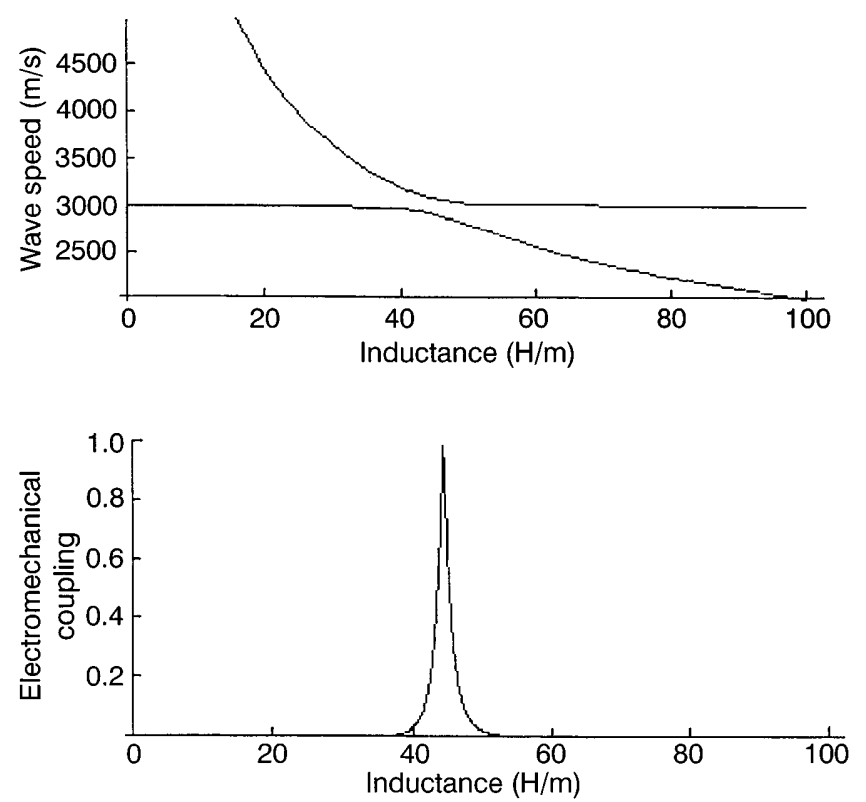

Fig. 5. Wave speeds and electromechanical coupling as functions of the line inductance (module 1: extensional problem) 
mechanical coupling. Our numerical results show that a relevant energy exchange occurs only in presence of too high impedance. In our opinion, this circumstance is due to the choice of an unsuitable spatial second order circuitry in the transmission line.

\section{2}

\section{Modal analysis: a clamped-clamped electromechanical beam}

We perform the modal analysis for Eqs. (35), adding to them the following boundary condition:

$$
\begin{array}{ll}
\mathbf{u}(0, t)=0 & \mathbf{u}\left(L_{B}, t\right)=0, \\
\gamma(0, t)=0 & \gamma\left(L_{B}, t\right)=0,
\end{array}
$$

where $L_{B}$ is the total length of the beam (we assume $L_{B}=2 \mathrm{~m}$ ). These boundary conditions physically mean that the beam is mechanically clamped and electrically insulated. We postpone to further investigations the study of more complex boundary conditions.

In order to lighten the presentation of the obtained results, as from the qualitative point of view they are similar for the first and the second modules, in the following we alternatively show plots concerning only one of them.

\subsection{1}

\section{Eigenfrequencies and eigenfunctions}

Although this does not mean any change in the numerical analysis, we assume, in this subsection, $\kappa_{R}=0$.

Numerical computation shows that it is possible to select pairs of eigenfunctions with electrical and mechanical modal shapes exchanged, as clearly shown by Fig. 6 .

In Fig. 7 the locus of eigenfrequencies is plotted in the plane of frequency and line inductance; for $\kappa_{L}=\overline{\kappa_{L}}$ the eigenvalues corresponding to the pairs of modal shapes determined in Fig. 6 are equal.

As one can also see from Fig. 5, in the neighborhood of $\overline{\kappa_{L}}$ the eigenfunctions show coupled electromechanical component; as a consequence, when $\kappa_{L} \simeq \overline{\kappa_{L}}$, for every eigenfunction the elastic deformation energy (relative to displacement modal form) and the electric capacitive

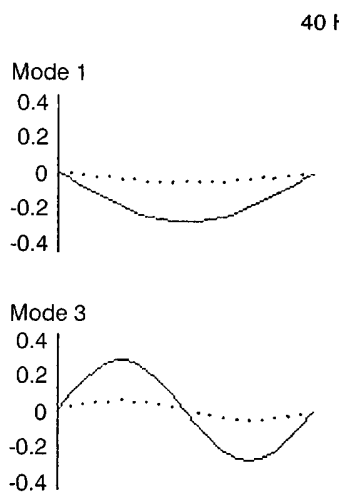

$40 \mathrm{H} / \mathrm{m}$
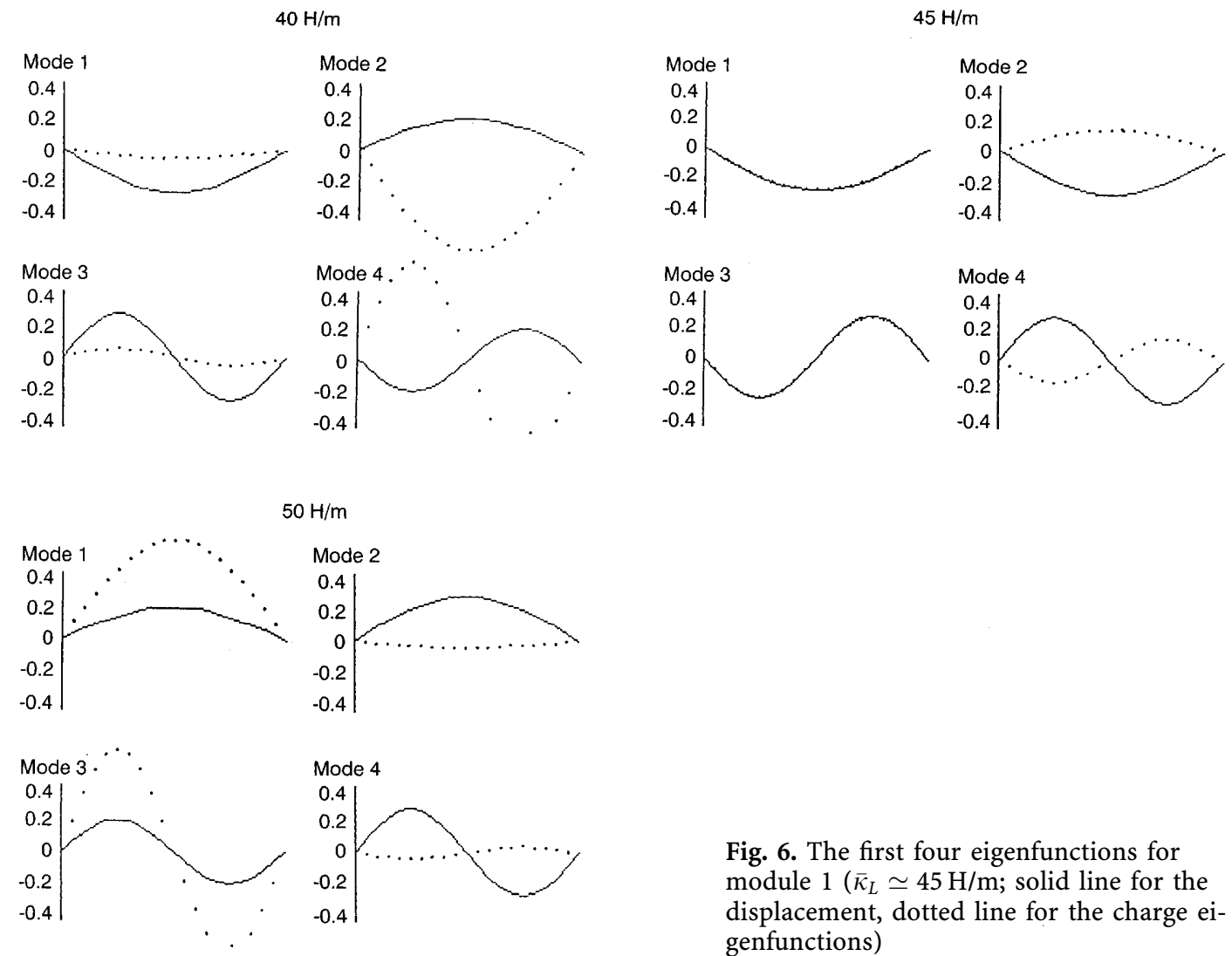

Fig. 6. The first four eigenfunctions for module $1\left(\bar{\kappa}_{L} \simeq 45 \mathrm{H} / \mathrm{m}\right.$; solid line for the displacement, dotted line for the charge eigenfunctions) 
energy (relative to charge modal form) are comparable; otherwise, for $\kappa_{L} \ll \overline{\kappa_{L}}$ or $K_{L} \gg \overline{\kappa_{L}}$, the eigenfunctions present either the mechanical or the electrical part sensibly greater than the other one.

\subsection{2}

Frequency response operator $\mathscr{H}_{j \omega}$

In this subsection, a dissipative line impedance is considered. A numerical evaluation of frequency response operator is obtained by a Fourier Transform of the FE discretized form of Eqs. (35). Let $\mathbf{H}(\omega)$ be the FE matrix representing $\mathscr{H}_{j \omega}$. In Fig. 8 we show the plot of $|\operatorname{det}[\mathbf{H}(\omega)]|$ for the twist angle-current problem for six different values of $\kappa_{L}$.

For the case considered in Fig. 8, the units are chosen so that $|\operatorname{det}[\mathrm{H}(0)]|=1$ and $\overline{\kappa_{L}} \simeq 330 \mathrm{H} / \mathrm{m}$.

The whole set of numerical simulations we have performed allows us to state the following qualitative results:

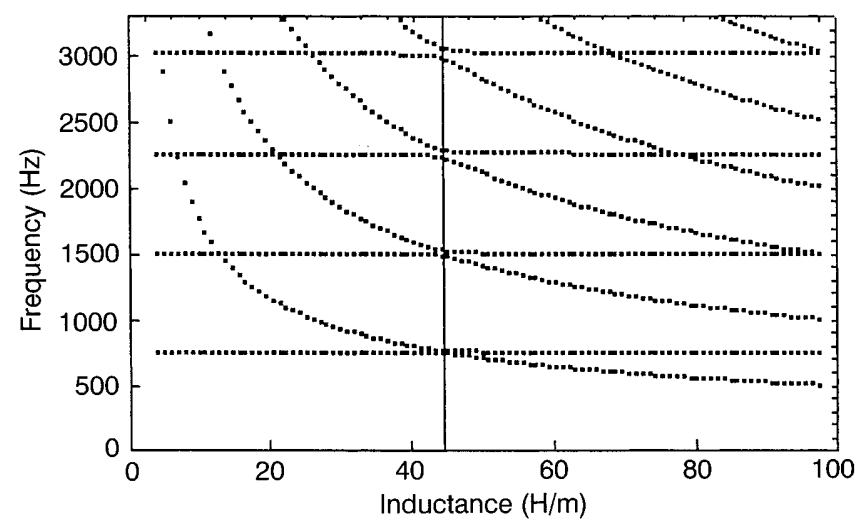

Fig. 7. Eigenfrequencies locus in the inductance frequency plane (module 1)
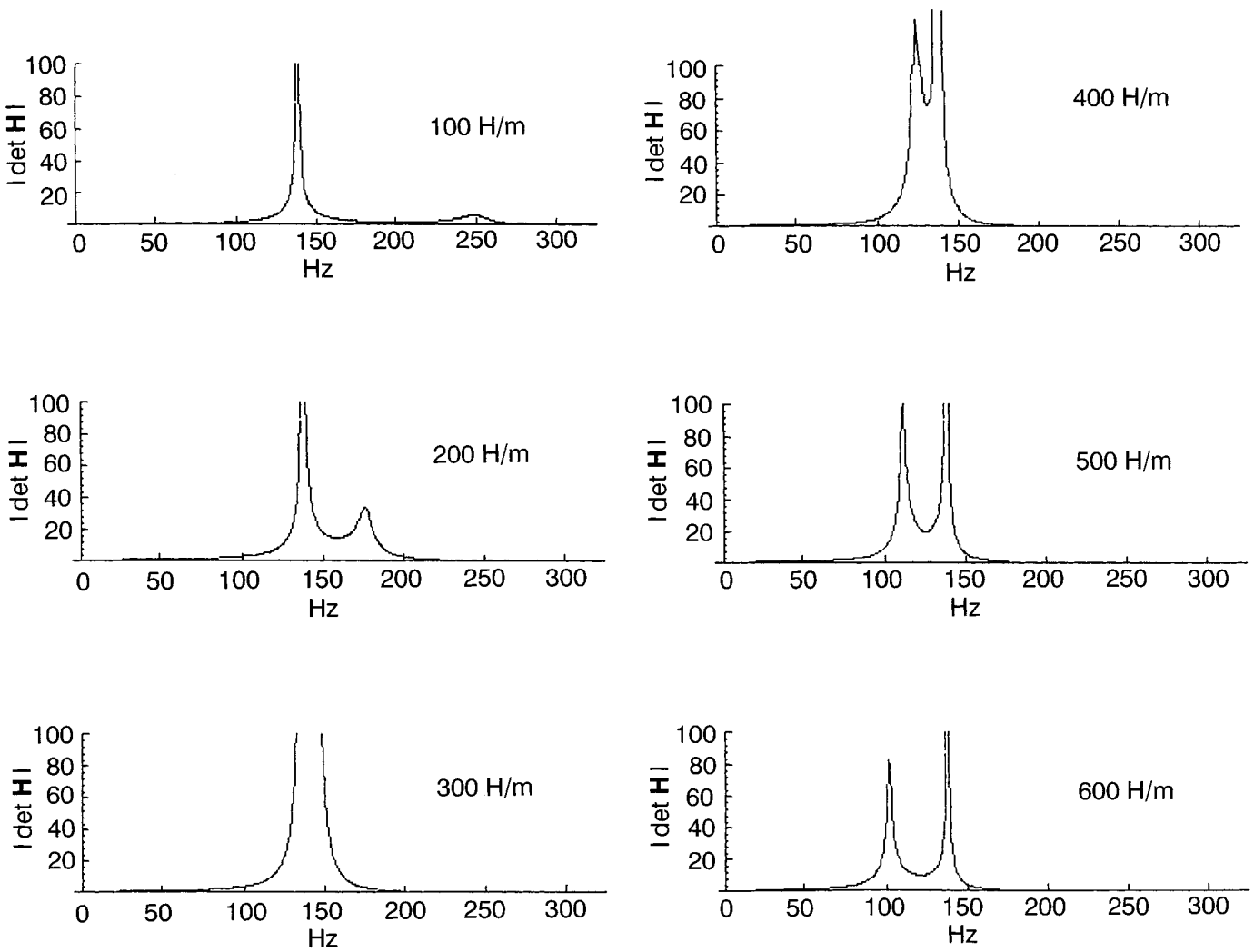

Fig. 8. Norm of $\operatorname{det}[H(\omega)]$ in the neighborhood of the first two eigenfrequencies (module $2: \bar{\kappa}_{L} \simeq 330 \mathrm{H} / \mathrm{m}$ ) 
- For $\kappa_{L} \ll \overline{\kappa_{L}}$ and $\kappa_{L} \gg \overline{\kappa_{L}}$, the plot of $|\operatorname{det}(\mathbf{H})|$ as a function of $\omega$ shows two distinct maxima; one of them is always finite and corresponds to the electric eigenfrequency, the other one is always infinite and corresponds to the mechanical eigenfrequency; this circumstance can be accounted for once one recalls the absence of any mechanical dissipation mechanism and the presence of an electric dissipative impedance.

- When $\kappa_{L} \simeq \overline{\kappa_{L}}$, the plot of $|\operatorname{det}(\mathbf{H})|$ has only one maximum; the considerations developed in the previous section indicate that in this case there is a relevant electromechanical energy exchange and coupling; this means that, for every forcing action, the system response shows comparable electrical and mechanical components.

- When $\kappa_{L} \simeq \overline{\kappa_{L}}$ the resonance frequency range increases its width with respect to the cases $\kappa_{L} \ll \overline{\kappa_{L}}$ and $\kappa_{L} \gg \overline{\kappa_{L}}$; apparently this could seem a drawback but the analysis of the following Sect. 4.3 shows that this is not the case. Indeed the resonance of the electrical signal allows for an efficient dissipation of a mechanical forcing action.

\section{3}

\section{An example of piezoelectromechanical damping}

In order to give a further test to the previous statements we implement a numerical simulation of a damping problem. More precisely, a time-evolution problem is studied: initial data are assumed to be given as a linear combination of the first two eigenfunctions.

Figure 9 clearly shows the electromechanical energy exchange in the case of non-dissipative impedance (dashed line) and the electrical damping of the mechanical vibration in the case of dissipative line (solid line).

In Fig. 10 are plotted the damping ratios of the first four modes as a function of line resistance. The damping ration $\delta$ is defined by the expansion:

$x(t)=x_{0} e^{-\delta t} e^{j \omega t}$

so that: $x(\bar{t})=x_{0} / e$ for $\bar{t}=\delta^{-1}$. There is a critical value for resistance for which the mechanical damping ratio attains its maximum (see curves $1 \mathrm{~m}$ and $2 \mathrm{~m}$ ).

In fact, Fig. 10 shows that for any mode there is a branching value $\tilde{\kappa}_{R}$ beyond which modal shapes are characterized by either a mechanical or an electrical dominant component (respectively, curves $1 \mathrm{~m}-2 \mathrm{~m}$ or $1 \mathrm{e}-2 \mathrm{e}$, where the first digit refers to mode frequency); this can be physically interpreted as follows: even when the modal coupling controlled by $\kappa_{L}$ is maximum, the choice of $\kappa_{R}$ can avoid the flowing of mechanical into electrical energy.
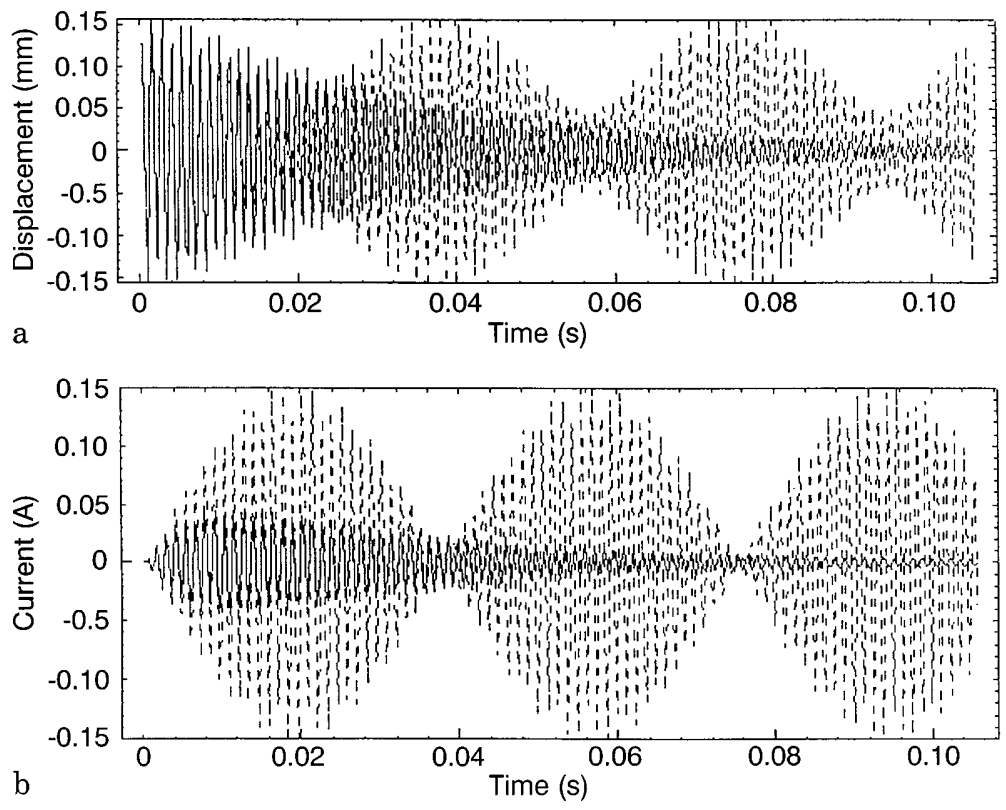

Fig. 9a,b. Time evolutions for axial displacement-current fields. $\left(\kappa_{L}=\overline{\kappa_{L}}\right.$; dashed line for $\kappa_{R}=0$, solid line for $\kappa_{R} \neq 0$ ) 


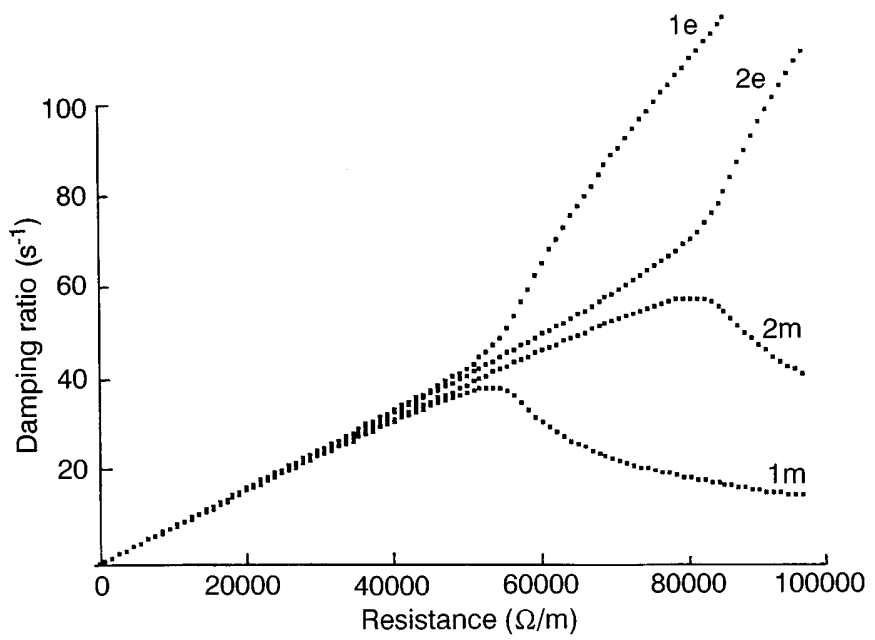

Fig. 10. Modal damping ratio vs. line resistance (module 2)

In Fig. 11 are plotted the damping ratios of the first four bending-charge modes for module 3 in the same range of line resistance, they are sensibly smaller than those shown in Fig. 10; this is essentially due to the low modal coupling between the bending beam and the second order transmission line presented. In order to obtain an efficient electric coupling for the bending beam we will introduce a spatially fourth order transmission line.

\section{5}

\section{Concluding remarks}

A modelling procedure for smart truss beams has been introduced essentially based on an identification in power; both the mechanical truss beam and electric transmission line have been described by continuum models in which PEM constitutive equations are expressed in terms of modular properties (generalizing [4] with method suggested in [2]).

The basic physical idea is to couple a truss beam with an electrical transmission line by means of piezoelectric elements which act respectively as bars and capacitances.

The piezoelectric stiffnesses and impedances have been derived by the tabular Voigt constants via a simple MATHEMATICA program (sketched in Appendix A), which implements results obtained in [7] and [8].

The continuum model leads to the equations of an internal variable continuum for $k_{r} \rightarrow \infty$ and of a microstructured continuum for $k_{R} \rightarrow \infty$.

Three different modules have been studied in order to investigate coupling phenomena between capacitive charge and, respectively, axial displacement, twist angle and bending. Our aim was to answer the following question: what happens when changing the line impedance (i.e. $\kappa_{L}$ and $\kappa_{R}$ )?

We found an interesting result: there is a critical line inductance value that maximizes the electromechanical modal coupling; this means a relevant exchange of energy between the beam and the electrical line and the possibility of an efficient control on the mechanical phenomena

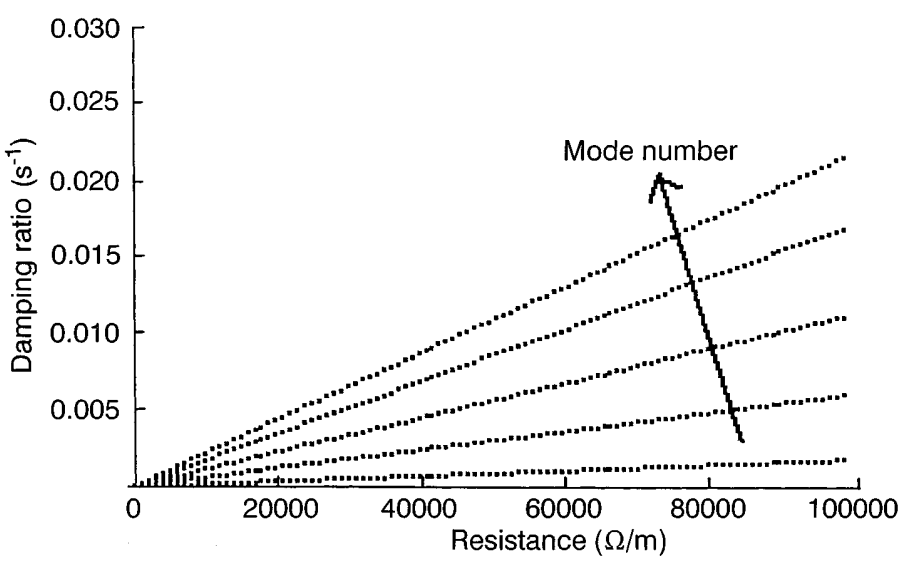

Fig. 11. Modal damping ratio vs. line resistance (module 3 ) 
acting on electric parameters. We only investigated a simple case of passive control in damping vibration, but several active control applications are conceivable, for instance acting on the terms $V$ and $v$ in Eqs. (35) and (36).

In particular, we obtain very high damping ratio in the case of extensional and torsional waves: distributing piezoelectric actuators along the beam and connecting them to form a transmission line proves to be a useful method for passive damping.

Unfortunately, in bending problems, the electromechanical coupling is a monotone increasing function of $\kappa_{L}$ and in technologically meaningful range of inductance is low; this is also shown by the damping ratio plots of Fig. 11: this circumstance is essentially due to the absence in the functional spectrum of the electrical line operator of the bending eigenfunctions (i.e. electric line and truss beam have different modal shapes) and will lead us to consider a spatially fourth order transmission line electrically paralleling a bending beam.

\section{Appendix A: Voigt constants}

In [1] are defined the following matrices representing the linear constitutive relation for a transversely isotropic piezoelectric (TIP) material:

$$
\begin{aligned}
\mathbf{s} & =\left(\begin{array}{cccccc}
s_{11} & s_{12} & s_{13} & 0 & 0 & 0 \\
s_{12} & s_{11} & s_{13} & 0 & 0 & 0 \\
s_{13} & s_{13} & s_{33} & 0 & 0 & 0 \\
0 & 0 & 0 & s_{44} & 0 & 0 \\
0 & 0 & 0 & 0 & s_{44} & 0 \\
0 & 0 & 0 & 0 & 0 & 2\left(s_{11}-s_{12}\right)
\end{array}\right) \\
\mathbf{d} & =\left(\begin{array}{cccccc}
0 & 0 & 0 & 0 & d_{15} & 0 \\
0 & 0 & 0 & d_{15} & 0 & 0 \\
d_{31} & d_{31} & d_{33} & 0 & 0 & 0
\end{array}\right) \\
\mathbf{k} & =\left(\begin{array}{ccc}
k_{11} & 0 & 0 \\
0 & k_{11} & 0 \\
0 & 0 & k_{33}
\end{array}\right) .
\end{aligned}
$$

There are ten independent material constants for such a material. The following Mathematica program allows to determine the only four coarse parameters $\left(k_{e e}, k_{e \rho}, k_{\rho e}, k_{\rho \rho}\right)$ of Eqs. (8) and (9) that are most technologically relevant, once the matrices $\mathbf{s}, \mathbf{d}$ and $\mathbf{k}$ are known.

$(* \operatorname{PZT} 5 *)$

$\mathrm{s} 11=16.4 ; \mathrm{s} 12=-5.74 ; \mathrm{s} 13=-7.22 ; \mathrm{s} 33=47.5 ; \mathrm{s} 44=47.5 ;\left(* 10^{\wedge}-12 *\right)$

$\mathrm{d} 15=584 ; \mathrm{d} 31=-172 ; \mathrm{d} 33=374 ;\left(* 10^{\wedge}-12 *\right)$

$\mathrm{k} 11=1730 ; \mathrm{k} 33=1700$;

$(*$ Experimental data $\longrightarrow$ Voigt Matrices $*)$

sigma $=$ Inverse $[\mathrm{s}]$;

$\mathrm{e}=\mathrm{d} \cdot$ Transpose[sigma] ;

eps $=$ eps0 k;

$\left(*\right.$ eps $0=$ permittivity of free space $\left.=8.85410^{\wedge}-12 *\right)$

$(*$ Voigt Matrices $\longrightarrow$ TIP material representation $*$ )

lambda $=\operatorname{sigma}[[1,1]]+\operatorname{sigma}[[1,2]] ; \mathrm{mu}=\operatorname{sigma}[[6,6]] / 2$;

alpha $1=\operatorname{sigma}[[4,4]] ;$ alpha $2=\operatorname{sigma}[[1,3]] ;$

alpha3 $=$ sigma $[[3,3]] / 2-$ alpha $2-$ alpha1;

beta $1=\mathrm{e}[[3,1]]-\mathrm{e}[[1,5]] ;$ beta2 $=-\mathrm{e}[[3,1]] ;$ beta $3=-\mathrm{e}[[3,3]]$;

gamma1 $=\operatorname{eps}[[1,1]] ;$ gamma2 $=\operatorname{eps}[[2,2]]$;

$(*$ TIP material representation $\longrightarrow[7] *)$

$\mathrm{A} 1=2($ alpha $1+$ alpha $2+$ alpha 3$)-$ alpha2 $\left.{ }^{\wedge}\right) /(\mathrm{mu}+$ lambda $) ;$

$\mathrm{A} 2=$ gamma $2+($ beta2 beta 1$) /(\mathrm{mu}+$ lambda $) ;$

$\mathrm{B} 1=$ beta $3-($ beta1 alpha 2$) /(\mathrm{mu}+$ lambda $)$;

$\mathrm{B} 2=-$ beta $3+($ beta2 alpha2 $) /(\mathrm{mu}+$ lambda $) ;$

$(*[7] \longrightarrow$ our actual definition $*)$

kee $=(\mathrm{A} 1-\mathrm{B} 1 \mathrm{~B} 2 / \mathrm{A} 2)$ Apzt; $(* \mathrm{Apzt}=\mathrm{PZT}$ cylinder section area $*)$ 
kep $=\mathrm{B} 1 / \mathrm{A} 2$

kpe $=-(\mathrm{B} 2 / \mathrm{A} 2)$ lpzt; $(*$ lpzt $=$ PZT cylinder length $*)$

$\mathrm{kpp}=(1 / \mathrm{A} 2) \mathrm{lpzt} / \mathrm{Apzt}$;

For the given PZT5 Voigt constants we obtain:

$\left(\begin{array}{ll}k_{e e} & k_{e \rho} \\ k_{\rho e} & k_{\rho \rho}\end{array}\right)=\left(\begin{array}{cc}24.085 \cdot 10^{9} \cdot A_{\mathrm{PZT}} & -1.1537 \cdot 10^{7} \\ -4.4664 \cdot 10^{8} \cdot l_{\mathrm{PZT}} & 7.9394 \cdot 10^{7} \cdot l_{\mathrm{PZT}} / A_{\mathrm{PZT}}\end{array}\right)$.

Appendix B: Continuum inertial actions

The inertial actions identified by the aforementioned procedure turn out to be the sum of two parts

$\int_{\varsigma 1}^{\varsigma 2}(\mathbf{b}, \mathbf{B}) \mathrm{d} \varsigma=\int_{\varsigma 1}^{\varsigma 2}(\tilde{\mathbf{b}}, \tilde{\mathbf{B}}) \mathrm{d} \varsigma+(\hat{\mathbf{b}}, \hat{\mathbf{B}})$

where $(\hat{\mathbf{b}}, \hat{\mathbf{B}})$ is a discrete measure concentrated in $\left\{\varsigma_{1}, \varsigma_{2}\right\}$ (for more details see [4]).

Moreover, the truss module inertial actions result from the sum over the set of the bars $\left(\mathscr{N}_{b}\right)$ and of bars constituting the diaphragms $\left(\mathscr{N}_{d}\right)$

$\tilde{\mathbf{b}}=\sum_{\mathscr{N}_{b}} \tilde{\mathbf{b}}_{b}+\sum_{\mathscr{N}_{d}} \tilde{\mathbf{b}}_{d}, \hat{\mathbf{b}}=\sum_{\mathscr{N}_{b}} \hat{\mathbf{b}}_{b}+\sum_{\mathscr{N}_{d}} \hat{\mathbf{b}}_{d}$

$\tilde{\mathbf{B}}=\sum_{\mathscr{N}_{b}} \tilde{\mathbf{b}}_{b}+\sum_{\mathscr{N}_{d}} \tilde{\mathbf{b}}_{d}, \hat{\mathbf{B}}=\sum_{\mathscr{N}_{b}} \hat{\mathbf{B}}_{b}+\sum_{\mathscr{N}_{d}} \hat{\mathbf{B}}_{d}$

Defining the following quantities $(x=b, d)$ :

$m_{x 0}=\int_{0}^{L_{x}} \rho_{x}(\xi)\left(1-\frac{\xi}{L_{x}}\right)^{2} \mathrm{~d} \xi$,

$m_{x 1}=\int_{0}^{L_{x}} \rho_{x}(\xi) \frac{\xi}{L_{x}}\left(1-\frac{\xi}{L_{x}}\right) \mathrm{d} \xi$,

$m_{x 2}=\int_{0}^{L_{x}} \rho_{x}(\xi)\left(\frac{\xi}{L_{x}}\right)^{2} \mathrm{~d} \xi$

the actions for a generic bar or diaphragm are given in terms of these integral mass quantities and of the coordinates $(\alpha, \beta)$ defined in Eq. (1)

$$
\begin{aligned}
\tilde{\mathbf{b}}_{b}= & -1 / L\left(m_{b 0}+2 m_{b 1}+m_{b 2}\right) \ddot{\mathbf{p}} \\
& -1 / L\left(\alpha_{b}^{+} m_{b 0}+\alpha_{b}^{+} m_{b 1}+\alpha_{b}^{-} m_{b 1}+\alpha_{b}^{-} m_{b 2}\right) \ddot{\mathbf{d}}_{2} \\
& -1 / L\left(\beta_{b}^{+} m_{b 0}+\beta_{b}^{+} m_{b 1}+\beta_{b}^{-} m_{b 1}+\beta_{b}^{-} m_{b 2}\right) \ddot{\mathbf{d}}_{3} \\
& +\left(-\alpha_{b}^{+} m_{b 1}+\alpha_{b}^{-} m_{b 1}\right) \ddot{\mathbf{d}}_{2}^{\prime} \\
& +\left(-\beta_{b}^{+} m_{b 1}+\beta_{b}^{-} m_{b 1}\right) \ddot{\mathbf{d}}_{3}^{\prime} \\
& +L / 4\left(m_{b 0}-2 m_{b 1}+m_{b 2}\right) \ddot{\mathbf{p}}^{\prime \prime} \\
& +L / 4\left(\alpha_{b}^{+} m_{b 0}-\alpha_{b}^{+} m_{b 1}-\alpha_{b}^{-} m_{b 1}+\alpha_{s} m_{b 2}\right) \ddot{\mathbf{d}}_{2}^{\prime \prime} \\
& +L / 4\left(\beta_{b}^{+} m_{b 0}-\beta_{b}^{+} m_{b 1}-\beta_{b}^{-} m_{b 1}+\beta_{b}^{-} m_{b 2}\right) \ddot{\mathbf{d}}_{3}^{\prime \prime}, \\
\hat{\mathbf{b}}_{b}= & 1 / 2\left(-m_{b 0}+m_{b 2}\right) \ddot{\mathbf{p}} \\
& +1 / 2\left(-\alpha_{b}^{+} m_{b 0}+\alpha_{b}^{+} m_{b 1}-\alpha_{b}^{-} m_{b 1}+\alpha_{b}^{-} m_{b 2}\right) \ddot{\mathbf{d}}_{2} \\
& +1 / 2\left(-\beta_{b}^{+} m_{b 0}+\beta_{b}^{+} m_{b 1}-\beta_{b}^{-} m_{b 1}+\beta_{b}^{-} m_{b 2}\right) \ddot{\mathbf{d}}_{3} \\
& +L / 4\left(-m_{b 0}+2 m_{b 1}-m_{b 2}\right) \ddot{\mathbf{p}}^{\prime} \\
& +L / 4\left(-\alpha_{b}^{+} m_{b 0}+\alpha_{b}^{+} m_{b 1}+\alpha_{b}^{-} m_{b 1}-\alpha_{b}^{-} m_{b 2}\right) \ddot{\mathbf{d}}_{2}^{\prime} \\
& +L / 4\left(-\beta_{b}^{+} m_{b 0}+\beta_{b}^{+} m_{b 1}+\beta_{b}^{-} m_{b 1}-\beta_{b}^{-} m_{b 2}\right) \ddot{\mathbf{d}}_{3}^{\prime},
\end{aligned}
$$




$$
\begin{aligned}
& \tilde{\mathbf{b}}_{d}=1 / L\left(-m_{d 0}-2 m_{d 1}-m_{d 2}\right) \ddot{\mathbf{p}} \\
& -1 / L\left(\alpha_{i} m_{d 0}+\alpha_{i} m_{d 1}+\alpha_{j} m_{d 1}+\alpha_{j} m_{d 2}\right) \ddot{\mathbf{d}}_{2} \\
& -1 / L\left(\beta_{i} m_{d 0}+\beta_{i} m_{d 1}+\beta_{j} m_{d 1}+\beta_{j} m_{d 2}\right) \ddot{\mathbf{d}}_{3} \\
& +L / 4\left(m_{d 0}+2 m_{d 1}+m_{d 2}\right) \ddot{\mathbf{p}}^{\prime \prime} \\
& +L / 4\left(\alpha_{i} m_{d 0}+\alpha_{i} m_{d 1}+\alpha_{j} m_{d 1}+\alpha_{j} m_{d 2}\right) \ddot{\mathbf{d}}_{2}^{\prime \prime} \\
& +L / 4\left(\beta_{i} m_{d 0}+\beta_{i} m_{d 1}+\beta_{j} m_{d 1}+\beta_{j} m_{d 2}\right) \ddot{\mathbf{d}}_{3}^{\prime \prime} \text {, } \\
& \hat{\mathbf{b}}_{d}=-L / 4\left(m_{d 0}+2 m_{d 1}+m_{d 2}\right) \ddot{\mathbf{p}}^{\prime} \\
& -L / 4\left(\alpha_{i} m_{d 0}+\alpha_{i} m_{d 1}+\alpha_{j} m_{d 1}+\alpha_{j} m_{d 2}\right) \ddot{\mathbf{d}}_{2}^{\prime} \\
& -L / 4\left(\beta_{i} m_{d 0}+\beta_{i} m_{d 1}+\beta_{j} m_{d 1}+\beta_{j} m_{d 2}\right) \ddot{\mathbf{d}}_{3}^{\prime}, \\
& \tilde{\mathbf{B}}_{b}=\mathbf{d}_{2} \wedge\left[-1 / L\left(\alpha_{b}^{+} m_{b 0}+\alpha_{b}^{+} m_{b 1}+\alpha_{b}^{-} m_{b 1}+\alpha_{b}^{-} m_{b 2}\right) \ddot{\mathbf{p}}\right. \\
& -1 / L\left(\alpha_{b}^{+2} m_{b 0}+2 \alpha_{b}^{+} \alpha_{b}^{-} m_{b 1}+\alpha_{b}^{-} m_{b 2}\right) \ddot{\mathbf{d}}_{2} \\
& -1 / L\left(\alpha_{b}^{+} \beta_{b}^{+} m_{b 0}+\alpha_{b}^{-} \beta_{b}^{+} m_{b 1}+\alpha_{d} \beta_{b}^{-} m_{b 1}+\alpha_{b}^{-} \beta_{b}^{-} m_{b 2}\right) \ddot{\mathbf{d}}_{3} \\
& +\left(\alpha_{b}^{+} m_{b 1}+\alpha_{b}^{-} m_{b 1}\right) \ddot{\mathbf{p}}^{\prime} \\
& +\left(-\alpha_{b}^{-} \beta_{b}^{+} m_{b 1}+\alpha_{b}^{+} \beta_{b}^{-} m_{b 1}\right) \ddot{\mathbf{d}}_{3}^{\prime} \\
& +L / 4\left(\alpha_{b}^{+} m_{b 0}-\alpha_{b}^{+} m_{b 1}-\alpha_{b}^{-} m_{b 1}+\alpha_{b}^{-} m_{b 2}\right) \ddot{\mathbf{p}}^{\prime \prime} \\
& +L / 4\left(\alpha_{b}^{+2} m_{b 0}-2 \alpha_{b}^{+} \alpha_{b}^{-} m_{b 1}+\alpha_{b}^{-} m_{b 2}\right) \ddot{\mathbf{d}}_{2}^{\prime \prime} \\
& \left.+L / 4\left(\alpha_{b}^{+} \beta_{b}^{+} m_{b 0}-\alpha_{b}^{-} \beta_{b}^{+} m_{b 1}-\alpha_{d} \beta_{b}^{-} m_{b 1}+\alpha_{b}^{-} \beta_{b}^{-} m_{b 2}\right) \ddot{\mathbf{d}}_{3}^{\prime \prime}\right] \\
& +d_{3} \wedge\left[-1 / L\left(\beta_{b}^{+} m_{b 0}+\beta_{b}^{+} m_{b 1}+\beta_{b}^{-} m_{b 2}\right) \ddot{\mathbf{p}}\right. \\
& -1 / L\left(\alpha_{b}^{+} \beta_{b}^{+} m_{b 0}+\alpha_{b}^{-} \beta_{b}^{+} m_{b 1}+\alpha_{d} \beta_{b}^{-} m_{b 1}+\alpha_{b}^{-} \beta_{b}^{-} m_{b 2}\right) \ddot{\mathbf{d}}_{2} \\
& -1 / L\left(\beta_{b}^{+2} m_{b 0}+2 \beta_{b}^{+} \beta_{b}^{-} m_{b 1}+\beta_{b}^{-} m_{b 2}\right) \ddot{\mathbf{d}}_{3} \\
& +\left(\beta_{b}^{+} m_{b 1}+\beta_{b}^{-} m_{b 1}\right) \ddot{\mathbf{P}}^{\prime} \\
& +\left(\alpha_{b}^{-} \beta_{b}^{+} m_{b 1}-\alpha_{b}^{+} \beta_{b}^{-} m_{b 1}\right) \ddot{\mathbf{d}}_{2}^{\prime} \\
& +L / 4\left(\beta_{b}^{+} m_{b 0}-\beta_{b}^{+} m_{b 1}-\beta_{b}^{-} m_{b 1}+\beta_{b}^{-} m_{b 2}\right) \ddot{\mathbf{p}}^{\prime \prime} \\
& +L / 4\left(\alpha_{b}^{+} \beta_{b}^{+} m_{b 0}-\alpha_{b}^{-} \beta_{b}^{+} m_{b 1}-\alpha_{d} \beta_{b}^{-} m_{b 1}+\alpha_{b}^{-} \beta_{b}^{-} m_{b 2}\right) \ddot{\mathbf{d}}_{2}^{\prime \prime} \\
& \left.+L / 4\left(\beta_{b}^{+2} m_{b 0}-2 \beta_{b}^{+} \beta_{b}^{-} m_{b 1}+\beta_{b}^{-2} m_{b 2}\right) \ddot{\mathbf{d}}_{3}^{\prime \prime}\right], \\
& \hat{\mathbf{B}}_{b}=d_{2} \wedge\left[1 / 2\left(-\alpha_{b}^{+} m_{b 0}-\alpha_{b}^{+} m_{b 1}+\alpha_{b}^{-} m_{b 1}+\alpha_{b}^{-} m_{b 2}\right) \ddot{\mathbf{p}}\right. \\
& +1 / 2\left(-\alpha_{b}^{+2} m_{b 0}+\alpha_{b}^{-2} m_{b 2}\right) \ddot{\mathbf{d}}_{2} \\
& +1 / 2\left(-\alpha_{b}^{+} \beta_{b}^{+} m_{b 0}+\alpha_{b}^{-} \beta_{b}^{+} m_{b 1}-\alpha_{d} \beta_{b}^{-} m_{b 1}+\alpha_{b}^{-} \beta_{b}^{-} m_{b 2}\right) \ddot{\mathbf{d}}_{3} \\
& +L / 4\left(-\alpha_{b}^{+} m_{b 0}+\alpha_{b}^{+} m_{b 1}+\alpha_{b}^{-} m_{b 1}-\alpha_{b}^{-} m_{b 2}\right) \ddot{\mathbf{p}}^{\prime} \\
& +L / 4\left(-\alpha_{b}^{+2} m_{b 0}+2 \alpha_{b}^{+} \alpha_{b}^{-} m_{b 1}-\alpha_{b}^{-} m_{b 2}\right) \ddot{\mathbf{d}}_{2}^{\prime} \\
& \left.+L / 4\left(-\alpha_{b}^{+} \beta_{b}^{+} m_{b 0}+\alpha_{b}^{-} \beta_{b}^{+} m_{b 1}+\alpha_{d} \beta_{b}^{-} m_{b 1}-\alpha_{b}^{-} \beta_{b}^{-} m_{b 2}\right) \ddot{\mathbf{d}}_{3}^{\prime}\right] \\
& +d_{3} \wedge\left[1 / 2\left(-\beta_{b}^{+} m_{b 0}-\beta_{b}^{+} m_{b 1}+\beta_{b}^{-} m_{b 1}+\beta_{b}^{-} m_{b 2}\right) \ddot{\mathbf{p}}\right. \\
& +1 / 2\left(-\alpha_{b}^{+} \beta_{b}^{+} m_{b 0}-\alpha_{b}^{-} \beta_{b}^{+} m_{b 1}+\alpha_{d} \beta_{b}^{-} m_{b 1}+\alpha_{b}^{-} \beta_{b}^{-} m_{b 2}\right) \ddot{\mathbf{d}}_{2} \\
& +1 / 2\left(-\beta_{b}^{+2} m_{b 0}+\beta_{b}^{-2} m_{b 2}\right) \ddot{\mathbf{d}}_{3} \\
& +L / 4\left(-\beta_{b}^{+} m_{b 0}+\beta_{b}^{+} m_{b 1}+\beta_{b}^{-} m_{b 1}-\beta_{b}^{-} m_{b 2}\right) \ddot{\mathbf{p}}^{\prime} \\
& +L / 4\left(-\alpha_{b}^{+} \beta_{b}^{+} m_{b 0}+\alpha_{b}^{-} \beta_{b}^{+} m_{b 1}+\alpha_{d} \beta_{b}^{-} m_{b 1}-\alpha_{b}^{-} \beta_{b}^{-} m_{b 2}\right) \ddot{\mathbf{d}}_{2}^{\prime} \\
& \left.+L / 4\left(-\beta_{b}^{+2} m_{b 0}+2 \beta_{b}^{+} \beta_{b}^{-} m_{b 1}-\beta_{b}^{-} m_{b 2}\right) \ddot{\mathbf{d}}_{3}^{\prime}\right] \text {, }
\end{aligned}
$$




$$
\begin{aligned}
\tilde{\mathbf{B}}_{d}= & \mathbf{d}_{2} \wedge\left[-1 / L\left(\alpha_{i} m_{d 0}+\alpha_{i} m_{d 1}+\alpha_{j} m_{d 1}+\alpha_{j} m_{d 2}\right) \ddot{\mathbf{p}}\right. \\
& -1 / L\left(\alpha_{i}^{2} m_{d 0}+2 \alpha_{i} \alpha_{j} m_{d 1}+\alpha_{j}^{2} m_{d 2}\right) \ddot{\mathbf{d}}_{2} \\
& -1 / L\left(\alpha_{i} \beta_{i} m_{d 0}+\alpha_{j} \beta_{i} m_{d 1}+\alpha_{i} \beta_{j} m_{d 1}+\alpha_{j} \beta_{j} m_{d 2}\right) \ddot{\mathbf{d}}_{3} \\
& +L / 4\left(\alpha_{i} m_{d 0}+\alpha_{i} m_{d 1}+\alpha_{j} m_{d 1}+\alpha_{j} m_{d 2}\right) \ddot{\mathbf{P}}^{\prime \prime} \\
& +L / 4\left(\alpha_{i}^{2} m_{d 0}+2 \alpha_{i} \alpha_{j} m_{d 1}+\alpha_{j}^{2} m_{d 2}\right) \ddot{\mathbf{d}}_{2}^{\prime \prime} \\
& \left.+L / 4\left(\alpha_{j} \beta_{i} m_{d 0}+\alpha_{j} \beta_{i} m_{d 1}+\alpha_{i} \beta_{j} m_{d 1}+\alpha_{j} \beta_{j} m_{d 2}\right) \ddot{\mathbf{d}}_{3}^{\prime \prime}\right] \\
& +\mathbf{d}_{3} \wedge\left[-1 / L\left(\beta_{i} m_{d 0}+\beta_{i} m_{d 1}+\beta_{j} m_{d 1}+\beta_{j} m_{d 2}\right) \ddot{\mathbf{P}}\right. \\
& -1 / L\left(\alpha_{i} \beta_{i} m_{d 0}+\alpha_{j} \beta_{i} m_{d 1}+\alpha_{i} \beta_{j} m_{d 1}+\alpha_{j} \beta_{j} m_{d 2}\right) \ddot{\mathbf{d}}_{2} \\
& -1 / L\left(\beta_{i}^{2} m_{d 0}+2 \beta_{i} \beta_{j} m_{d 1}+\beta_{j}^{2} m_{d 2}\right) \ddot{\mathbf{d}}_{3} \\
& +L / 4\left(\beta_{i} m_{d 0}+\beta_{i} m_{d 1}+\beta_{j} m_{d 1}+\beta_{j} m_{d 2}\right) \ddot{\mathbf{P}}^{\prime \prime} \\
& +L / 4\left(\alpha_{i} \beta_{i} m_{d 0}+\alpha_{j} \beta_{i} m_{d 1}+\alpha_{i} \beta_{j} m_{d 1}+\alpha_{j} \beta_{j} m_{d 2}\right) \ddot{\mathbf{d}}_{2}^{\prime \prime} \\
& \left.+L / 4\left(\beta_{i}^{2} m_{d 0}+2 \beta_{i} \beta_{j} m_{d 1}+\beta_{j}^{2} m_{d 2}\right) \ddot{\mathbf{d}}_{3}^{\prime \prime}\right] \\
\hat{\mathbf{B}}_{d}= & \mathbf{d}_{2} \wedge\left[-L / 4\left(\alpha_{i} m_{d 0}+\alpha_{i} m_{d 1}+\alpha_{j} m_{d 1}+\alpha_{j} m_{d 2}\right) \ddot{\mathbf{P}}^{\prime}\right. \\
& -L / 4\left(\alpha_{i}^{2} m_{d 0}+2 \alpha_{i} \alpha_{j} m_{d 1}+\alpha_{j}^{2} m_{d 2}\right) \ddot{\mathbf{d}}_{2}^{\prime} \\
& \left.-L / 4\left(\alpha_{i} \beta_{i} m_{d 0}+\alpha_{j} \beta_{i} m_{d 1}+\alpha_{i} \beta_{j} m_{d 1}+\alpha_{j} \beta_{j} m_{d 2}\right) \ddot{\mathbf{d}}_{3}^{\prime}\right] \\
& +\mathbf{d}{ }_{3} \wedge\left[-L / 4\left(\beta_{i} m_{d 0}+\beta_{i} m_{d 1}+\beta_{j} m_{d 1}+\beta_{j} m_{d 2}\right) \ddot{\mathbf{P}}^{\prime}\right. \\
& -L / 4\left(\alpha_{i} \beta_{i} m_{d 0}+\alpha_{j} \beta_{i} m_{d 1}+\alpha_{i} \beta_{j} m_{d 1}+\alpha_{j} \beta_{j} m_{d 2}\right) \ddot{\mathbf{d}}_{2}^{\prime} \\
& \left.-L / 4\left(\beta_{i}^{2} m_{d 0}+2 \beta_{i} \beta_{j} m_{d 1}+\beta_{j}^{2} m_{d 2}\right) \ddot{\mathbf{d}}_{3}^{\prime}\right] . \\
& -1
\end{aligned}
$$

\section{Appendix C: Linearization}

The linearization of the continuum model inertial actions shown before is obtained with a simple Mathematica symbolic program that provides to calculate $(\mathbf{b}, \mathbf{B})$ via the substitution

$$
\begin{aligned}
p & \rightarrow P+\varepsilon\left(u \mathbf{D}_{1}+v \mathbf{D}_{2}+w \mathbf{D}_{3}\right), \\
\mathbf{d}_{1} & \rightarrow \mathbf{D}_{1}+\varepsilon\left(\vartheta_{3} \mathbf{D}_{2}+\vartheta_{2} \mathbf{D}_{3}\right), \\
\mathbf{d}_{2} & \rightarrow \mathbf{D}_{2}+\varepsilon\left(\vartheta_{1} \mathbf{D}_{3}-\vartheta_{3} \mathbf{D}_{1}\right), \\
\mathbf{d}_{3} & \rightarrow \mathbf{D}_{3}-\varepsilon\left(\vartheta_{2} \mathbf{D}_{1}+\vartheta_{1} \mathbf{D}_{2}\right),
\end{aligned}
$$

where $(u, v, w)$ are the displacement field components and $\left(\vartheta_{1}, \vartheta_{2}, \vartheta_{3}\right)$ are the parameters of the rotation field $\mathbf{R}$

linealist $=\{$

$\mathrm{D}[\mathrm{p}[\mathrm{s}, \mathrm{t}],\{\mathrm{t}, 2\}] \rightarrow(\mathrm{u} * \mathrm{D} 1+\mathrm{v} * \mathrm{D} 2+\mathrm{w} * \mathrm{D} 3)$,

$\mathrm{D}[\mathrm{d} 1[\mathrm{~s}, \mathrm{t}],\{\mathrm{t}, 2\}] \rightarrow(+$ teta3 $* \mathrm{D} 2+$ teta $2 * \mathrm{D} 3)$,

$\mathrm{D}[\mathrm{d} 2[\mathrm{~s}, \mathrm{t}],\{\mathrm{t}, 2\}] \rightarrow(-$ teta3 $* \mathrm{D} 1+$ teta $1 * \mathrm{D} 3)$,

$\mathrm{D}[\mathrm{d} 3[\mathrm{~s}, \mathrm{t}],\{\mathrm{t}, 2\}] \rightarrow(-$ teta $1 * \mathrm{D} 2-$ teta $2 * \mathrm{D} 3)$,

$\mathrm{D}[\mathrm{p}[\mathrm{s}, \mathrm{t}],\{\mathrm{t}, 2\},\{\mathrm{s}, 1\}] \rightarrow($ us $* \mathrm{D} 1+\mathrm{vs} * \mathrm{D} 2+\mathrm{ws} * \mathrm{D} 3)$,

$\mathrm{D}[\mathrm{d} 1[\mathrm{~s}, \mathrm{t}],\{\mathrm{t}, 2\},\{\mathrm{s}, 1\}] \rightarrow(+$ teta3s $* \mathrm{D} 2+$ teta2s $* \mathrm{D} 3)$,

$\mathrm{D}[\mathrm{d} 2[\mathrm{~s}, \mathrm{t}],\{\mathrm{t}, 2\},\{\mathrm{s}, 1\}] \rightarrow(-$ teta3s $* \mathrm{D} 1+$ teta1s $* \mathrm{D} 3)$,

$\mathrm{D}[\mathrm{d} 3[\mathrm{~s}, \mathrm{t}],\{\mathrm{t}, 2\},\{\mathrm{s}, 1\}] \rightarrow(-$ teta1s $* \mathrm{D} 2$-teta2s $* \mathrm{D} 1)$,

$\mathrm{D}[\mathrm{p}[\mathrm{s}, \mathrm{t}],\{\mathrm{t}, 2\},\{\mathrm{s}, 2\}] \rightarrow($ uss $* \mathrm{D} 1+\mathrm{vss} * \mathrm{D} 2+$ wss $* \mathrm{D} 3)$,

$\mathrm{D}[\mathrm{d} 1[\mathrm{~s}, \mathrm{t}],\{\mathrm{t}, 2\},\{\mathrm{s}, 2\}] \rightarrow(+$ teta3ss $* \mathrm{D} 2+$ teta2 $2 \mathrm{ss} * \mathrm{D} 3)$,

$\mathrm{D}[\mathrm{d} 2[\mathrm{~s}, \mathrm{t}],\{\mathrm{t}, 2\},\{\mathrm{s}, 2\}] \rightarrow(-$ teta3ss $* \mathrm{D} 1+$ teta $1 \mathrm{ss} * \mathrm{D} 3)$,

$\mathrm{D}[\mathrm{d} 3[\mathrm{~s}, \mathrm{t}],\{\mathrm{t}, 2\},\{\mathrm{s}, 2\}] \rightarrow(-$ teta1ss $* \mathrm{D} 2$-teta2ss $* \mathrm{D} 1)\}$;

$\mathrm{b}=\mathrm{b} /$.linealist;

$\mathrm{B}=\mathrm{B} /$.linealist;

In a similar way is obtained the linearization of the contact actions $(\mathbf{s}, \mathbf{S})$. 


\section{References}

1. Eringen, A. C.; Maugin, G. A.: Electrodynamics of Continua I, New York: Springer 1990

2. Maugin, G. A.: The method of virtual power in continuum mechanics: application to coupled fields. Acta Mech. 35 (1980) 1-70

3. Maugin, G. A.: Material Inhomogeneities in Elasticity, London: Chapman \& Hall 1993

4. Di Carlo, A.; Rizzi, N.; Tatone, A.: Continuum modelling of beam-like latticed truss: identification of the constitutive functions for the contact and inertial actions. Meccanica 25 (1990) 168-174

5. Noor, A. K.; Andersen, C. M.: Analysis of beam-like lattice trusses. Comput Methods Appl. Mech. Eng. 2 (1979) 53-70

6. Abrate, S.: Continuum modelling of latticed structures. Shock Vib. Digest 17 (1985) 15-21

7. dell' Isola, F.; Rosa, L.: Saint-Venant Problem in Linear Piezoelasticity. In: Proceedings of SPIE Conference on "Smart Materials and Structures", pp. 24-29. San Diego, California, 1996

8. dell' Isola, F.; Rosa, L.: Almansi-type boundary conditions for electric potential inducing flexure in linear piezoelectric beams. to appear in Continuum Mechanics and Thermodynamics.

9. Vidoli, S.: Strutture spaziali flessibili: modellazione e simulazione orientate al controllo. Tesi di Laurea, Università degli Studi di Roma "La Sapienza", 1995

10. Teresi, L.: Analisi modale di travature reticolari modulari. Tesi di Laurea, Università degli Studi di Roma "La Sapienza", 1990

11. Guran, A.; Inman, D. J.: Wave motion, intelligent structures and nonlinear mechanics. Singapore: World Scientific 1995

12. Near, C. D.: Piezoelectric actuator technology. In: Proceedings of SPIE Conference on "Smart Materials and Structures", pp. 24-29. San Diego, California 1996

13. Culshaw, B.: Smart structures and materials. Boston: Artech House 1996 